\title{
Virtual Machine Migration in Vehicular Cloud for Service Continuity
}

\author{
Fuad Nagi Shahrah \\ Faculty member in the Department of Computer \\ Network and Information Technology \\ Faculty of Engineering, AL-Qalam University for \\ Humanities and Applied Sciences, Yemen
}

\author{
Fekri Mohammed Ahmed Abduljalil \\ Associate Professor and the Head of Computer \\ Science Department \\ Faculty of Education and Applied Science - \\ Khawlan, University of Sana'a, Yemen
}

\begin{abstract}
Vehicular Cloud (VC) is an emerging technology where multiple vehicles form a cloud to share their abundant resources and carry out a heavy job in a cooperative manner. By using $\mathrm{VC}$, each vehicle can run several $\mathrm{VC}$ applications which require heavy resources by creating virtual devices in vehicles during the execution of services by vehicles in a geographical zone chosen by the user relying on a Service-Level Agreement (SLA). The vehicle may be out of the service zone and that may cause a disruption in service and loss of data what makes the provider need to connect to another vehicle which gets wasted time. In this paper, the previous proposal which presents migrate Virtual Machines (VM) from the vehicle to any node in the service zone is reviewed. Then, a vehicle selection method based on the vehicle's remaining time in the service zone is proposed. After that, this proposal is implemented in Python, a simulation program. The experiment results showed that the process of selection and VM migrate avoids data loss during service interruption. It also avoids wasting time when re-selecting a vehicle and establishing a connection with another vehicle.
\end{abstract}

\section{Keywords}

Virtual machine, Virtual machine migration, Vehicular cloud, Cloud computing, Total migration time, Vehicular ad hoc networks, Cloud zone.

\section{INTRODUCTION}

Recently, technology has developed rapidly and researchers have not been able to cope with these rapid developments. One of the most important topics that researchers have taken care of is cloud computing. According to National Institute of Standards and Technology (NIST), "Cloud computing is a model that allows ubiquity, convenience and on-demand access to a set of configurable shared resources and it can be quickly delivered with minimal management effort on the clients part"[1].

The NIST definition lists five essential characteristics of cloud computing: on-demand self-service, broad network access, resource pooling, rapid elasticity or expansion, and measured service [2]. Taking into consideration the huge volume of cloud computing and its applications, it is necessary to transfer from traditional cloud computing to ad hoc network cloud computing. Therefore, a new cloud computing model called VANET-Cloud is proposed and applied to the vehicle self-organizing networks. [3].

Vehicle networks serve as one of the most important enabling technologies required to implement a myriad of applications related to vehicles. At the present, it has witnessed a shift from traditional vehicular ad hoc networks (VANETS) to VANET Cloud network. At the beginning, the concept of Vehicular Clouds (VCs) has been firstly introduced in [4-8] by Professor Olariu and his co-workers.

Vehicular ad hoc networks (V ANETs) offer an interconnected communication and computing platform; they also utilize vehicles equipped with embedded computer systems, i.e. computing, storage and networking resources. Thus, most vehicles nowadays are produced with these facilities. These resources mostly remain underutilized.

Future VANET deployments are expected to better utilize of these resources by consolidating them in a cloud platform, whereby each connected vehicle will act as a mobile physical host in a data center [10].

Due to the mobility of the vehicle, when the vehicle is moving on the road, it will often move from one cloud to another frequently. Therefore, cloud services must be transferred from one cloud site to another.

In cloud-based vehicle networks, vehicles are regarded as the resource of the consumers and the providers where they can use several cloud services that are different from other tools such as the Roadside infrastructure and ITS central server. [11]

Vehicle Cloud Network (VCN) offers a wide range of applications such as, Video Surveillance in Urban Zones, Bandwidth Management, Real-time Navigation, Remote Traffic Management [12], and other applications such as File Sharing, Custom Local Shopping Advertisement and Vehicular Relay Chat between users.

Due to the characteristics of high mobility, handling VMM issues in cloud-based vehicle networks is essential to keep cloud services running [13].

In the cloud, computing is used as the main enabling technology to organize all physical resources in the vehicle network. However, deploying the cloud in the vehicle network still has to face many challenges. The next few lines will summarize some of the main challenges [13]:

- Continuity of Cloud Services: The mobility of vehicles causes dynamic changes in the network topology. Frequent changes may disrupt ongoing cloud services. Therefore, cloud resources management should be carefully designed. 
- Accessibility of Cloud Services: Vehicle networks are essentially hybrid networks that mix different types of wireless mobile communication systems. The cloud architecture is preferred to provide interfaces to support different types of access modes.

- Connectivity. The management and control of network connections among vehicles and between vehicles and network infrastructure are the most important issues for VANET communication. The main challenge in designing vehicle communication is to provide good delay performance under the constraints of vehicle speed, high dynamic topology and channel bandwidth [14].

- Mobility. Mobility that is the norm for vehicular networks makes the topology change quickly. In addition, the mobility patterns of vehicles on the same road will show a strong correlation, and the authors address the idea that mobility plays a key role in vehicular protocol design and modeling .

In this paper, this problem is studied and a method for continuity of cloud vehicle services in data center is proposed through the virtual machine live migration technology that allows the virtual machine to move from one vehicle to another.

The rest of this paper is organized as follows; Section 2 examines Cloud Computing with Cloud Based Vehicular Network architecture. Section 3 highlights the Virtual Machine Migration in Cloud Based Vehicular with a proposed method to keep cloud services running on. Finally, Section 4 presents a conclusion and future directions.

\section{PREVIOUS STUDIES}

In [3], Samiur Arif, Stephan Olariu, Jin Wang, Gongjun Yan, Weiming Yang and Ismail Khalil considered that in typical international airports, people usually travel and leave their cars at the airport for many days and this provides a wide range of cars that could function as a data center inside the airport.

That is why they expected scenarios to connect the vehicles with each other via the Internet and the cloud as well as provide the vehicles with energy and then connect them to the central server of the airport in order to be able to schedule resources and assign the tasks of different vehicles on the cloud network.

To complete the work they did, it was necessary to obtain an expected number of vehicles presented in the parking lot and how long to wait in the airport parking.

In [11], Oanh Tran Thi Kim, Nguyen Dang Tri, VanDung Nguyen, and Choong Seon They conducted a study on the movement of vehicles on the road where the vehicles are moving frequently between the clouds. Therefore; the cloud service must move from one cloud site to another. As a result, they put forward the concept of VMM as a solution to use the vehicle resources efficiently. They presented a complete diagram of VMM scenarios in the vehicle network and all the scenarios were considered. In light of that, many research challenges and open problems were presented to the deployment and implementation of VMM in the field of cloud computing and network vehicles.

In [13], T. K. Refaat, B. Kantarci, and H. T. Mouftah proposed a scheme called: Vehicular Virtual Machine

\section{Migration (VVMM).}

This study seeks to find out an effective mechanism for the changes of data centers topology and the heterogeneity of the components of the cloud network including RSU.

The study was divided into three modes. The first one is the mode before the vehicle leaves the RSU coverage.

The second mode is migration at the lowest workload on vehicles and the data center.

The third mode is after migrating the virtual machine at the lowest workload and within the geographic boundaries. But in this case, the migration is expected with previous knowledge.

In [14], Rong Yu, Yan Zhang, Huimin Wu, Periklis Chatzimisios, and Shengli Xie They took into consideration the fact that the movement of vehicles is one of the major challenges of the continuity of the service and to solve such problem, they proposed a relay mechanism for Virtual Machine and then they worked on designing a migration strategy that improves the efficiency of data transmission during the process of migration in the vehicle network.

In [15], Rong Yu ,Yan Zhang , Stein Gjessing , Wenlong $\mathrm{Xia}$, Kun They studied virtual machine migration and network resource allocation for the sake of efficiently managing resources in vehicle network within the cloud.

The results of the above study indicated that virtual machine migration due to vehicle mobility is solved based on a resource reservation scheme.

Although the above studies were significant and have contributed a lot in the field of cloud-based vehicular network, there is still a problem for the users of the services and applications of vehicle networks which is the interruption of service and the need to reconnect again. Hence, in the study at hand, a proposed solution will be provided to continue the service in the network of vehicles so that services and applications won't be interrupted when the connection to the vehicle is lost or when the vehicle is out of the contact zone.

This proposal is using virtual machines migration to continue the service in the vehicle network.

\section{SYSTEM MODEL}

In this section, the system model, assumptions, problem statement and design objectives are introduced as follows:

The proposed architecture in this paper is shown in Figure 1 , consists of the following entities:

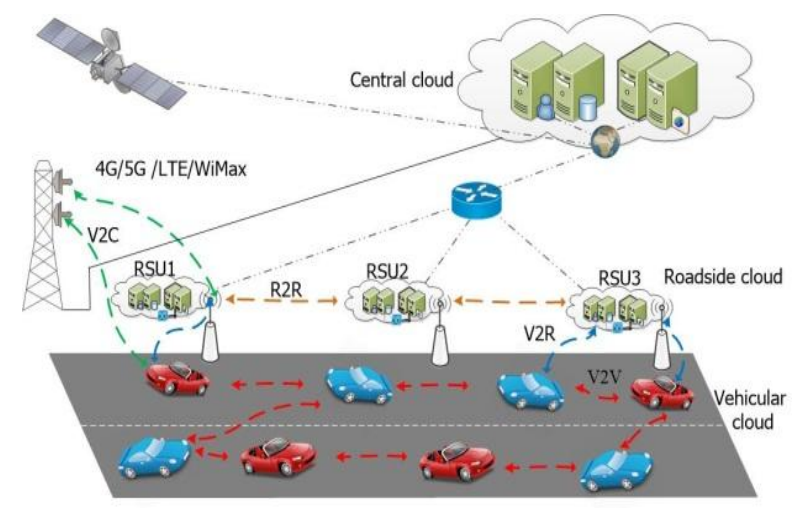

Figure 1: Cloud Based Vehicular Network Architecture 
- The Vehicular Cloud: A local cloud that is established within a group of cooperative vehicles. The vehicle-to-vehicle network (i.e., VANET) is formed through $\mathrm{V} 2 \mathrm{~V}$ communication. The vehicles in a group are regarded as mobile cloud sites and jointly create a vehicle cloud.

- Roadside Cloud: A local cloud which is organized within a group of adjacent roadside units. The cloud on the roadside allows the vehicles to use V2R communications for access.

- Central Cloud: An established cloud within a set of dedicated servers on the Internet. Vehicles will access the central cloud via V2R or Any type of wireless/ cellular communication.

- Cellular network cloud: The cellular network is a network of communication among vehicles and the cellular communication system by long distance communications. E.g., 4G/5G cellular.

- Satellite network cloud: A network for connecting the vehicles to the satellite to reach the cloud in the absence of other networks.

- Cloud service: any service provided to users on demand from the server of the cloud computing provider through the Internet, rather than the service provided from the company's own local server. cloud services are designed to access to applications and provide easy they are scalable at resources, and services, and are fully managed by cloud service providers. For example, real-time navigation with computing resource sharing. And video surveillance and storage resource sharing.

- Cloud zone: is the zone that we need to cover a specific service agreement between the service provider and the vehicle network with the user who needs this service where this zone varies by service level agreement. They are also limited by the range RSU or the range of the network coverage.

- Service provider: in the Vehicle Cloud, the leading applications are safe driving, urban sensing, content distribution, mobile advertising and intelligent transportation. For example, vehicles pick up information via sensors (e.g., congestion, pavement conditions, surrounding cars, environment video clips, advertisements, etc.). They organize, exchange and keep the data local since local relevance and sheer volume of this data making Internet upload unattractive. Other vehicles or internet users can search for the data in the Vehicle Cloud with proper indexing and scoping. There will also be significant computing on this cloud. For instance, computation of the full urban congestion picture; computation of the urban pollution map; collaborative reconstruction of pictures/video in accident or crime scenes; coordinated identification of possible terrorist threats; etc.

- The available services in a vehicular cloud are divided into two main categories: (1) Infrastructure as a Service (IaaS) that describes the available physical resources. (2) Software as a Service (SaaS) that describes VANET adapted applications such as infotainment or traffic and comfort driving applications.
This study has several assumptions, the most important of which are stated as follows:

- The time in which the connection between the data center and the vehicle is established; it is greater than the migration time in the vehicle network.

- The connection between the vehicle and the data center is secure and subjected to a secure verification process and no one can eavesdrop on this connection to steal the data.

- If the vehicle leaves the service zone before Virtual machine migration, the data will be lost.

- A service provider will have to migrate VM to RSU if the resources owned by the predefined vehicle are insufficient.

In the vehicle network, continuous movement is a feature that can be exploited to take advantage of network vehicle sources, but this continuous movement may be constitute a challenge for users. When the user needs a service in zone, for example, video capture, important data will be lost when interruption and therefore it may lose money as a result of this interruption and this is what happens in car accidents, especially insurance companies when shooting accidents.

The movement of the vehicle leads to the departure of the vehicle from the service zone and to disconnect the communication with the vehicle. Thus, the vehicle needs to restore contact with another vehicle to provide the user with data from the service zone. The data center wastes time when searching for another vehicle to get the data, so the problem here is wasting time and losing data which are the two factors that concern the user in the first order.

There is loss of time and data due to leaving the vehicle from the service zone. In this paper, a proposal for continuity of data flow and exploitation of vehicle survival in the zone will be presented.

The proposed solution is based on the exploitation of virtual machine migration technology in the vehicle network. When the vehicle enters the zone, the virtual machine is created in each vehicle connected to it. The virtual machine provides the data center with the data it needs. When the vehicle is expected to leave the service zone, the virtual machine is moved to another vehicle or to RSU near the zone to save data when no vehicle is present for the migration.

\section{PROPOSED METHOD}

In This section, the basic Virtual machine migration Is explained. After that the basic idea behind our service will be proposed, and then the proposal of VMM in vehicular clouds will described in detail.

\subsection{Virtual Machine Migration}

Before talking about the scenario chosen to implement the proposal, the virtual machine migration in vehicular network in cloud computing (VMM-VCC) will be talked about.

Virtual machine migration is the process of moving a virtual machine between physical hosts where OS and all applications are migrated as one integrated unit between physical hosts.

Virtual machine migrations in cloud computing 
environment are generally composed of the following:

(1) Determining the source host machine from which some virtual machines are to be removed. (2) Determining the destination host machine to which some virtual machines will be moved. (3) Choosing one of the appropriate types of migration (such as static migration, dynamic migration, or mixed migration). (4) Carrying out the virtual machine migration. (5) Deleting the corresponding data associated with the removed virtual machine on the source host.

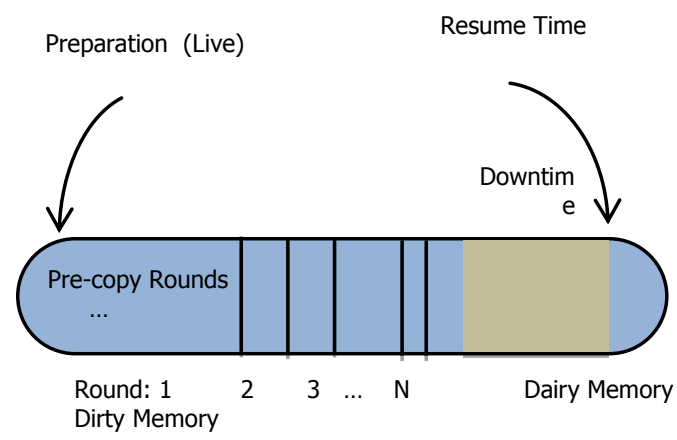

(a) Pre-Copy Timeline
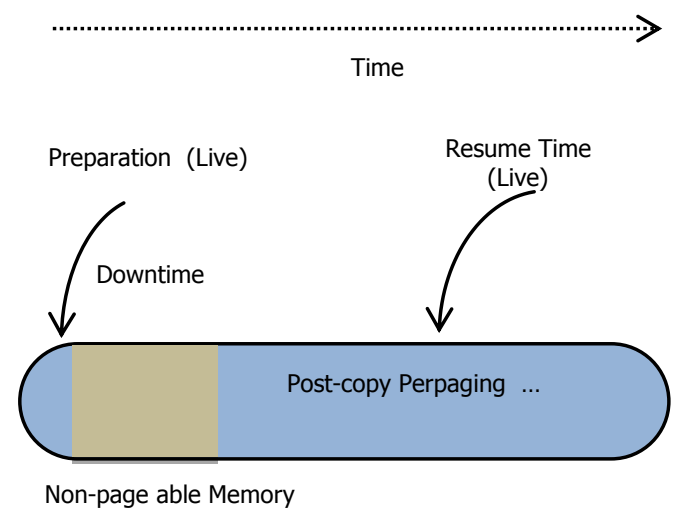

(b) Post-copy Timeline

\section{Figure 2. Timeline of Pre copy vs. Post copy migration}

\section{Virtual Machine Migration methods are divided into two types show in Figure 2:}

1. Hot (live) migration: Virtual machine keeps running while migrating and does not lose its status.

The user does not feel the interruption of the service in hot (live) migration and in this case, the virtual machine state migration is transferred.

The state consists of its memory contents and local file system. Local file system needs not to be transferred.

Live migration is a migration during which the VM seems to be responsive all the time from clients' perspective. In the current study, in addition to the previous goals, achieving this goal is looked forward to.

Live migration is classified into two steps; (i) Controlling is switched to the destination. (ii) Data Transferring (memory/disk) to the destination [16].
There are two major approaches; Pre-Copy and Post-Copy memory migration shown in Figure 2.

(a) Pre-copy: There are two phases in Pre-copy approach; Warm-up phase and Stop-and-Copy phase. In warm up VM memory migration phase, the hypervisor copies all the memory pages from source to destination while the VM is still running on the source. If some memory pages change during memory copy process - dirty pages, they will be recopied until the rate of recopied pages is not less than page dirtying rate. In Stop and Copy phase, the VM will be stopped in source and the remaining dirty pages will be copied to the destination and VM will be resumed in destination. A dirty page at the source is a memory page whose state is different from the destination. These pages should be copied to the destination

(b) Post-copy: It first suspends the migrating VM at the source, copies minimal processor state to the target node, resumes the virtual machine, and begins fetching memory pages over the network from the source.[17]

The following metrics are used to measure the performance of live migration:

i) Preparation- Here, resources are reserved on the destination which performed various operations.

ii) Downtime- Time during which the VM on the source host is suspended

iii) Resume- It does the instantiation of VM on the destination but with the same state as suspended source.

iv) Total time- The total time taken in completion of all these phases is called Total Migration time.

2. Cold (non-live) migration- The status of the VM loses and user can notice the interruption of the service.

In cold migration, first, VM is suspended then its state is transferred. Finally, VM is resumed at destination host.

Memory transfer is divided into three phases:

i) Push phase: Certain pages are pushed in advance to the new destination before the source virtual machine moves to destination. Some modified pages are resent to ensure consistency. ii) Stop and copy phase: It is a simple way in which source virtual machine is stopped at source and complete. Virtual machine is copied or moved to destination and then resumed it at destination. iii) Pull phase: In this phase, virtual machine starts execution on destination machine and if it requires a page that has not been copied, this page is pulled across the network from the source virtual machine.

\subsection{Basic Idea Behind VMM in VC}

One of the challenges that face the vehicle network is the interruption of the connection with the data center that happens in many cases for many reasons including leaving the vehicle from the contact zone where such leaving leads to previous data loss. Therefore, the reestablishment of communication is required, but the reestablishment process leads to a delay in service return process as it needs time.

In what follows, the proposed virtual machine migration 
in vehicular cloud for service continuity will be presented. One of such services can be represented by a regionspecific video service for the purpose of taking pictures of events in that zone and sending live video to servers for analysis and handling.

The vehicle can connect to the cloud through one of the interfaces on the vehicle such as a roadside link, radio waves, wimax or satellite to deliver the video service to the applications that require this service. The network of vehicles is always moving, so the video host will enter the service zone at a certain time and move within a certain time until the vehicle is leaving from the zone.

It is necessary to continue providing servers with live videos, because when the vehicle becomes out of the region, the service will be outage. Migration to VM that hosts this service in the local roadside servers before the vehicle leaves the zone is proposed. The virtual machine will be transferred from a host to another.

One of the main motivations of the migration process of virtual machines is the moving of resources in cloud vehicles. This process is very important where it is carried out for various purposes and the migration is an important event.

As stated above, there are four scenarios for the migration of virtual devices in the network of vehicle. In this paper, one of these scenarios that is, an inter-road migration closer to the vehicle network will be focused on.

\subsection{Virtual machine migration in VCC}

Vehicles are equipped with OBU (On-Board Unit) that consists of a computing device, a unique identification number, a range wireless interface, a radio transceiver, an event data recorder, front and rear sensors, a Global Positioning System (GPS) device, etc. This information will be used to track the Vehicles.

Commonly, a smart vehicle is equipped with the following devices and technologies: (i) a Central Processing Unit (CPU) that implements the applications and communication protocols. (ii) A wireless transceiver for data transmissions among vehicles (V2V) and from vehicles to RSUs (V2I). (iii) A Global Positioning Service (GPS) receiver for positioning and navigation services. (iv) Different sensors laying inside and outside the vehicle to measure various parameters (i.e., speed, acceleration, distance from neighboring vehicles, etc.). (v) An input/output interface for human interaction with the system [18].

A Virtual Machine (VM) is a simulation of a real or abstract machine. VM research has been mainly studied in computer networks. In [19], VM migration is considered for dynamic resource management in cloud environments. In [20], VM replication and scheduling are intelligently combined for VM migration across wide zone network environments. The study in [21] conducted a number of interesting experiments to compare several resource management schemes for VM migration.

Advancements in vehicular technology and Intelligent Transportation Systems (ITS) have provided new applications that demand complex computation and large storage. However, due to hardware and cost limitations, a single vehicle has limited computation and storage resources. Hence, it is difficult for an individual vehicle to efficiently support these applications [22].
On the other hand, these equipment are often untapped and can be better exploited by integrating them into a cloud platform, whereby each connected vehicle will act as a mobile physical host in a data center.

In a conventional cloud data center, physical servers are partitioned into multiple VM and users are assigned VMs so that they can use the same physical environment by being totally isolated from each other.

Improving the use of physical resources, reliability and energy efficiency are the most important reasons for migrating a virtual machine from one physical host to another where virtualization does the migration process for logical resources..

To perform a migration from one host to another, there are sequential steps to take advantage of virtual machine migration technology.

In this work, studying virtual machine migration techniques will not be focused on because it is not a concern in this proposal, but the main objective will be how to choose the appropriate vehicle. The steps of choosing such vehicle are ordered as follows and shown step by step in Figure 3:

\subsubsection{Cloud Zone Definition}

Cloud zone: A geographic zone with cloud service provider data centers. Users select one or several zones provided by service providers according to the need.

Service providers determine this zone according to the user needs and the range of network coverage available.

The SLA is a content of all the service zone details in terms of the geographical zone specified for the beginning and the end of the zone where services are available (i.e., direct imaging) as well as vehicle accessories, RSU and what price of each service which the user will pay. The zone that is worked on in this paper is a longitudinal zone.

\subsubsection{Vehicle Registration}

Nodes (i.e., vehicles and RSUs in the vicinity) in cloud zone often communicate through multiple routing. Each node sends broadcast messages to all nodes in cloud zone using the Federal Communications Commission-mandated (DSRC) or the Federal Communications Commission (FCC) to obtain information of vehicles or equipment located in that cloud zone. Each node sends location information by GPS (Global Positioning System) device to cloud zone [23]. Then, it can calculate the velocity based on timely communication of location from the other nodes. 


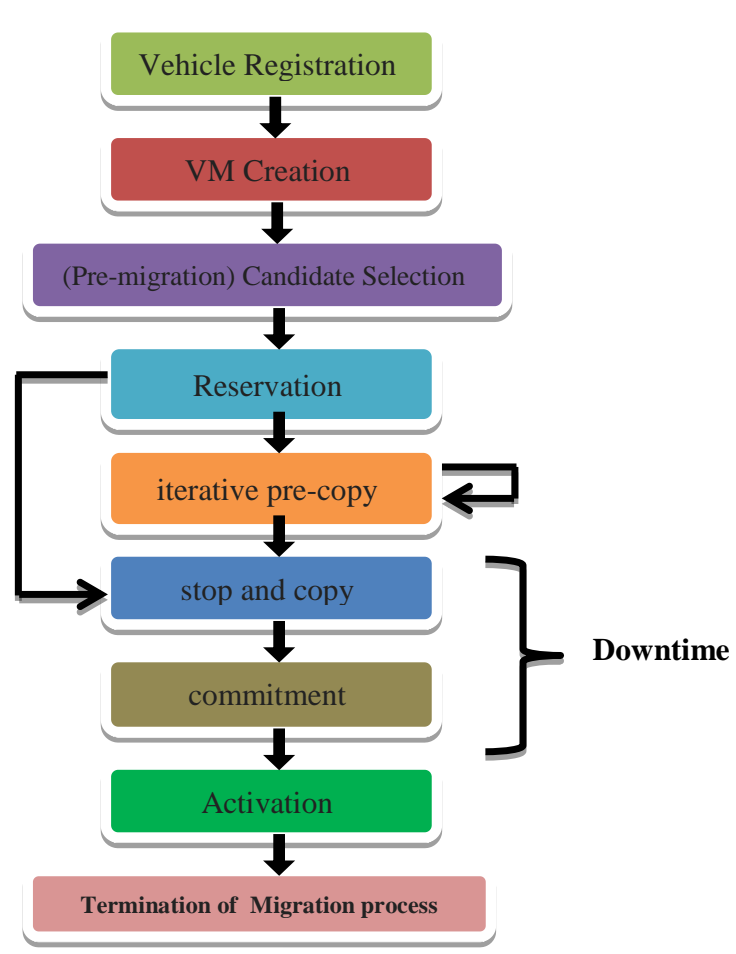

Figure 3: Steps in pre-copy Technique Migration

After the service provider communicates with the data center to obtain service from the cloud zone, the data center creates a database for that service with basic information about the cloud zone. In addition, any node enters the cloud zone and records its basic data including (chassis number, date of manufacture, vehicle accessory devices, sensors, vehicle equipment, data storage and computing and what services) are available on this vehicle.

A node between the node and the data center in the cloud zone is authenticated to create a secure connection where the vehicles with the access permission are allowed to connect to the data center to obtain information for any node in the zone.

They can communicate with each other to see which service can be used between those nodes (sharing files, exploiting resources).

The vehicle can perform tasks such as storing and browsing the Internet, exchanging data through servers in the network and sending and receiving information such as; weather, heat, accident locations, traffic jams and other information that a user may need for a particular zone through the vehicle sensor.

The connection continues and updates data between nodes and the data center in the region.

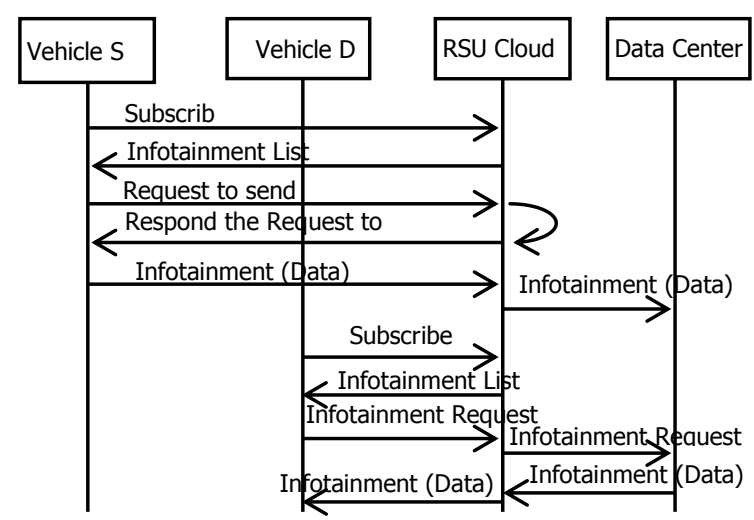

Figure 4: Sequence Diagram Communication between Vehicle and RSU and Data Center

The communication process is show in Figure 4, RSU (RSU Cloud Member) broadcasts a beacon message for vehicles entering the service area. All vehicles receive the guide's message. It is connected to RSU with IEEE 802.11 MAC.

Assuming that vehicle $\mathrm{S}$ would like to send the information message, you will subscribe to the RSU cloud then keep track of sending an information request message to the RSU cloud. Attached RSU will update the information list to the RSU cloud and respond to the requested message from vehicle $S$. Finally, the $S$ vehicle will send all information data to the attached RSU then RSU will redirect all data to the data center to retain the data. If the targeted $\mathrm{D}$ vehicle needs to receive information data, it will subscribe and wait to receive the information list message.

Vehicles wait for any service request coming from the data center. If the vehicle does not receive any request, data and information about that vehicle will be deleted after leaving the zone.

\subsubsection{VM Creation}

Depending on the definition of the vehicle network, it has a complete infrastructure and can perform different functions.

When the vehicle enters the service zone, a VM is creating a picture of the actual node that processes the services in the node, so that the vehicle is ready to request service.

The user requests the service from a provider by accessing the website of the available services. After the approval of the user request, the provider searches the database for vehicles that can meet the user's request to be contacted and creates a virtual device in the vehicle to host the service.

This happens in all vehicles that are added to the database to be ready for the migration.

4.3.4 Service request: (first vehicle selection) The service provider creates a connection to the service request from the vehicle that meets the needs and near the service start point. This is the first step in the service delivery process. The mechanism for selecting the vehicle for the first time conforms to the specifications. The last vehicle is also entered from the beginning of the service zone. Data when vehicle registration makes the last vehicle enter is the first vehicle to be nominated for service. 


\subsubsection{VM Migration Decision}

Depending on the SLA and the service requested by the user, some services require a continuous flow of data such as direct imaging of the zone if the user has recommended that the data is of great importance (priority).

When user loses some data at the time of interruption, cloud service provider shall make a decision on whether its corresponding VM shall be migrated or not [24].

We need some issues to decide whether to migrate or not and then to make the right decision. We must answer the necessary question of whether the decision to migrate the virtual machine is useful and can achieve the goal of deportation or not. The decision to migrate depends on some factors that would issue the deportation decision including:

- Loss of interest in the user data that can determine whether the data is an important task provided or the priority of this service on the basis of SLA agreed between the user and the service provider.

- Sources (storage, processing, etc.) of the current vehicle are not sufficient while working on the service and can refer to SLA to see whether the process allows provisioning of additional sources or not.

- Breakdown Current vehicle: In this case, there is no option. When the vehicle breakdown in the service zone, the immigration decision must be made.

When the cost of migration of VM into the vehicle network is more expensive than data or that the migration time is more than the time of connection with a new vehicle entering the service zone, it is a mistake to do VM migration.

In the current work, the remaining time was assumed to be greater than the time of the migration. It was also assumed that there is a mandatory point of migration for the migration of the VM. Before the arrival of the vehicle to the end plan in sufficient time, the decision must be made to migrate directly because the vehicle has reached the mandatory immigration zone.

Obviously, to migrate the VM, the image must be transferred from the source to the destination. The transfer cost is charged and the time required relays where the decision is based on the status of the VM and its data.

\subsection{6 (Pre-migration) Candidate Selection}

To select the right vehicle for the migration, there are two situations:

- The service provider: the service provider is responsible for selecting the appropriate vehicle and VM migration by the resource manager's.

In this case, the service provider will view the database and calculate the remaining time before the vehicle leaves the service zone as well as it will get alerts if the vehicle is breakdown or the service needs additional sources which are not owned by the current vehicle.

- The vehicle: the vehicle is responsible for selecting the appropriate vehicle for migration.

The current vehicle queries the information required for all vehicles in the service zone from the database and it can also get information by contacting other vehicles in the network vehicles. These data include the remaining time of vehicle in the service zone, Location of the vehicle and vehicle equipment (sources). Accordingly, the current vehicle can choose the appropriate vehicle for VM migration.

To select the appropriate vehicle for the migration process, the remaining time for all vehicles to reach the end point will be calculated. In this proposal, it will be assumed that the first case that the service provider is choosing the right vehicle since the vehicles may not have the right equipment to perform the calculations they need to choose the right vehicle as it is necessary to have a target high-efficiency node selection policy to migrate the VM to the appropriate host.

Depending on the calculations for the vehicle's location and the factors that will come, the data center looks at the database to select the appropriate vehicle for migration.

To speed up any future migration, the target host may be specified in advance and the resources required to receive the relay will be guaranteed and this actual host is determined by factors as follows [25]:

- Vehicle lifetime: the time taken by a chosen vehicle from the instance of observation before it leaves its current service zone.

- The position of vehicle: the position of the chosen vehicle from the beginning of the current service zone at the instance of observation. (A vehicle at the start of the service zone is more likely to have a larger lifetime before it leaves the service zone).

- Vehicles speed: the speed of the chosen vehicle at the instance of observation.

- Speed average of vehicles ahead in the same service zone at the instance of observation (A vehicle at a congested zone is more likely to decelerate and have a larger lifetime).

- The vehicle's moving path within the service zone.

- The host infrastructure as it can be chosen but cannot meet the requirements.

In this paper, the remaining time of the vehicle in the service zone will be depended on.

When the vehicle enters the service zone, it is added to the database as mentioned above and the vehicle that finally entered is in the highest order. Before calculating the remaining time of the vehicle in the service zone, the service provider will test the vehicle. Does this vehicle match the specifications required by the service? If the specifications match, the remaining time is calculated, otherwise the vehicle is ignored.

\subsubsection{Reservation}

A request is issued to migrate VM from Host A to Host B. Host B is selected and only Host A controls after confirming all the requirements as well as the service level agreement SLA and authenticates the migration by Host B and the reservation part of the available resources (i.e. CPU, Memory and Storage).

\subsubsection{Iterative pre-copy}

It has been concluded that there are many techniques that make the migration process extremely effective. However, during the migration, the number of duplicated pages that exceed the total time is increasing the migration time and 
the total downtime. Therefore, there is a need for an approach that reduces duplicate page transfers (which were modified again after they were transmitted to target host). Migration time and total downtime are key zones of improving the migration process.

In the process of migrating VM, it is important to migrate memory pages, especially in memory pages that are currently executing.

Fast migration can be achieved by compressing memory pages. In this mechanism, pages are compressed at the source using various compression techniques and decompressed at the destination.

Memory compression can reduce the amount of data transfer during the migration process. Using compression can also reduce the cost of transferring virtual machine memory, storage content and service downtime during migration [26].

\subsubsection{Stop and copy}

At this phase, the source is stopped and the remaining pages (dirty pages) are copied. If the dirty pages in this iteration are less than the iteration- 1 that preceded it, the running $\mathrm{OS}$ instance at $\mathrm{A}$ is suspended and its network traffic will be redirected to B. As described earlier, CPU state and any remaining inconsistent memory pages are then transferred. At the end of this stage, there is a consistent suspended copy of the VM at both A and B. The copy at A is still considered to be primary and is resumed in case of failure.

\subsubsection{Commitment}

Host B indicates that host A has successfully received a consistent OS image. Host A confirms that this message is a promise of the migration transaction. It can now discard the original VM, and host B becomes the primary host.

\subsubsection{Activation}

The migrated VM on B is now activated. The code will run after the migration to reconnect the device driver with the new computer and advertise the moved IP address.

\subsubsection{Termination of Migration process}

After the migration process of the virtual machine is completed from source host to target host and the vehicle leaves the cloud zone, the vehicle needs to delete the temporary files from the resources for any new request in the process of processing to providing services

In electronic devices, you get unexpected errors. One of these errors is the failure of the migration process. If something happens during the migration process and the virtual machine is not migrated and the vehicle exits from the service zone, immediate migration is made to the nearest RSU then returns the VM to the RSU in the service zone and the service provider will restore service in the service zone.

\section{IMPLEMENTATION \& EVALUATION}

The proposed algorithms have been simulated with Python, which is topology. The mobility model, VM initialization, establishes contact, sends service request and ensures that service requirements are met and calculate the remaining time for vehicles in the zone.

Two algorithms have been proposed to implement the proposal:
- The first algorithm is candidating selection algorithm for VM migration.

- The second algorithm is VM migration in vehicle Network.

Candidate selection algorithm for VM migration:

1. Input VC[Id, SPEED v, ZONE, $\partial x$, loc ]

2. Output VC in (DB)

3. For Each VC In $D B$

4. If $V C d \neq m i g$ req Then

5. Ignore $\mathrm{VC}_{\mathrm{d}}$

6. Else

7. Calculate $\partial t$ where

8. $\partial t=\partial x / \mathrm{v}$ where

9. $\partial x 1=x-\partial x$

10. Add $\partial t$ to VC DB Table .

11. Endif

12. End for

13. Sort VC info table by $\partial t$ desc

14. Return first_Record

15. End

Service continuity algorithm for VM in the vehicle network

1. input: VC, RSU, DC ,DB, zone, loc

2. Output: VM in zone.

3. Beginning

4. For Each VC enters the zone

$5 . \quad$ Registration $\mathrm{VC}$

6. Create VM in VC

7. $\quad$ Add VC to DB

8. $\mathrm{VCs}=$ call candidate selection algorithm

9. End for

10. Request service from VCs

11. Start service.

12. if VCs stop \{

13. Get request from VCs

14. $\mathrm{VCd}=$ call candidate selection algorithm

15. Do migration \}

16. Elseif $\{$ loc $=x-\partial x=20$

17. $\mathrm{VCd}=$ call candidate selection algorithm

18. Do migration \}

19. Endif

20. Delete data from VCs

21. End

Simulation is used to evaluate the performance of each of the proposed algorithms based on several metrics by proposing inputs and analyzing it. The work can be illustrated as follows:

-The service Zone in which the vehicles move may be in two directions. In this work, one direction only will be adopted because there will be a repetition of the other direction.

$$
\text { Zone }=\text { np.random.randint }(1,3, y)
$$

It is defined by the zone in the two different directions and it is between the numbers $(1,3)$ where $(y)$ is the number of vehicles in the specified Zone.

The service zone was divided into sub-regions: (20\%) for each region.

Deportation will be requested if the vehicle reaches $80 \%$ of the total service Zone because this point is the compulsory migration point for the vehicle. The data is 
transferred from VC source to VC destination or to the RSU.

In this work, the sub- Zone was divided for searching for vehicles in the first Zone. If it does not find it, it moves to the other Zone and this makes the simulation faster than it searches in all Zones.

$$
\text { for des in range(20, 80, 20): }
$$

So the vehicles are arranged according to their presence near the vehicle entry point in the database that was previously created in the data center of the service Zone.

- The time periods for the number of vehicles in the service Zone were divided into three sections:

- The first section: when the zone is not crowded.

- The second section: when the vehicles are in the normal state.

- The third section: when the vehicles are crowded.

- So that it was suggested that the number of vehicles are arranged as follows according to sections $(12,8$, 4)

$$
\text { vm_in_str }=[12,8,4]
$$

Vehicle speed according to traffic rules in the service zone within cities which ranges between $(20,80)$ kilometers per hour.

$$
\text { speed }=\text { np.random.randint }(20,80, y)
$$

Test the vehicle's conformity to the requirements of the relay and this was done by some random selection functions, so that if the number (1) the vehicle meets the requirements otherwise (0) does not meet the requirements.

mig_req $=n p \cdot$ random.choice $(2,1 * y, p=[0.3,0.7])$

\section{Simulation Metrics and Stages}

- The time of the vehicle stays in the service zone.

The vehicle's survival time can be calculated in the service zone if we know the following for $\mathrm{VC}_{\mathrm{d}}$ :

- Space of service zone: The space is recognized by the service provider according to the SLA.

- The speed of the vehicle when entering the zone at any time via vehicle sensors.

- The time of entry of the vehicle Service zone.

\section{- Time to traverse the zone from the starting point} to the end point

The mathematical formula can be used to calculate the time to traverse the zone from the starting point to the end point according to the information that the vehicle sends to the data center.

$$
\begin{gathered}
\partial t=\frac{\partial x}{v} \\
\mathrm{v}=\text { speed }, \partial x=\text { distance and } \partial t=\text { time }
\end{gathered}
$$

\section{- Remaining distance from a particular point}

When the vehicle is moving in the service zone, we need to find the remaining distance from a particular point, so this formula is used:

$$
\partial x 1=x-\partial x
$$

$\partial x 1$ is distance from any point to the zone end , $x$ is

\section{all zones and $\partial x$ distace \\ from start or any point in zone to $d x 1$}

\section{- Calculate the remaining distance}

To find out the time needed to complete the remaining distance, use the following formula:

$$
\partial t=\frac{\partial x 1}{v}
$$

After making calculations in the data center and updating the database which contains all information about vehicles, the vehicles are arranged in the database according to the time remaining in the zone that meets the requirements for service. The vehicle with the most time remaining is to be chosen for the VM migration. When simulating, the simulation result will be as follows:

Table (1): vehicles in the database

\begin{tabular}{|c|c|c|c|c|c|c|}
\hline \multicolumn{7}{|c|}{ Database of Virtual Machines: } \\
\hline & ID & Speed & Zone & Xa & Data & Mig_Req \\
\hline 0 & 0 & 56 & 1 & 22 & 0 & 1 \\
\hline 1 & 1 & 40 & 1 & 16 & 0 & 1 \\
\hline 2 & 2 & 62 & 1 & 61 & 0 & 1 \\
\hline 3 & 3 & 23 & 2 & 23 & 0 & 1 \\
\hline 4 & 4 & 72 & 1 & 32 & 0 & 1 \\
\hline 5 & 5 & 63 & 2 & 93 & 0 & 0 \\
\hline 6 & 6 & 57 & 2 & 7 & 0 & 0 \\
\hline 7 & 7 & 70 & 1 & 78 & 0 & 1 \\
\hline
\end{tabular}

Table (1) shows the two Zones $(1,2)$. The first Zone No. (1) will be chosen.

Mig_Req the extent to which the vehicle meets the requirements of the VM migration, $\partial x$ is location the vehicle in the zone.

Table (1) shows the number of vehicles in the zone No. (5). These vehicles fulfill all the conditions, but they vary in their location in this Zone.

Table (2) vehicles in the zone (1)

We Have 5 VMs in owr zone(1)

Frist $5^{\text {th }}$ are:

\begin{tabular}{|c|c|c|c|c|c|c|}
\hline & ID & Speed & Zone & Xa & Data & Mig_Req \\
\hline 0 & 0 & 25 & 1 & 48 & 0 & 1 \\
\hline 1 & 1 & 53 & 1 & 31 & 0 & 1 \\
\hline 3 & 3 & 52 & 1 & 68 & 0 & 1 \\
\hline 4 & 4 & 43 & 1 & 38 & 0 & 1 \\
\hline 6 & 6 & 26 & 1 & 35 & 0 & 1 \\
\hline
\end{tabular}

Table (2) contains the number of vehicles that were found in the service zone No. (1) and that meets the requirements. The first 5 vehicles were brought in. If we have more than 5 vehicles, only the first (5) vehicles will appear and take the advantage of the time in the process of calculating the remaining time and the remaining distance of the vehicle in the service zone. 
Looking at Table (1), we see that vehicle No. (7) with location $\mathrm{Xa}=(78)$ was not selected in Table (2) because its location is nearer to the final point. As a result, a filter is made for the vehicles to obtain the first 5 vehicles that are nearer to the starting point.

Table (3) Search in the Service Zone

\begin{tabular}{|c|c|c|c|c|c|c|}
\hline \multicolumn{7}{|c|}{ We Have 5 VMs in with Xa $<20 \%$ : } \\
\hline \multicolumn{7}{|c|}{ Empty Data Frame } \\
\hline \multicolumn{7}{|c|}{ Columns: [ID, Speed, Zone, Xa, Data, Mig_Req ] } \\
\hline \multicolumn{7}{|c|}{ Index: [ ] } \\
\hline \multicolumn{7}{|c|}{ We Have 5 VMs in with $\mathrm{Xa}<40 \%$ : } \\
\hline & ID & Speed & Zone & $\mathrm{Xa}$ & Data & Mig_Req \\
\hline 1 & 1 & 53 & 1 & 31 & 0 & 1 \\
\hline 4 & 4 & 43 & 1 & 38 & 0 & 1 \\
\hline 6 & 6 & 26 & 1 & 35 & 0 & 1 \\
\hline
\end{tabular}

Table (3) shows the search process which is as follows:

The sub-zones $(20 \%)$ are searched first then move to the next until they find the appropriate vehicle to be selected and start the migration.

In the above table, there is no vehicle in the first part, $20 \%$ of the service zone and it is moved to second part $40 \%$.

After moving from the first part $20 \%$ to the second part $40 \%, 3$ vehicles were found and there the comparison process between the vehicles in terms of speed is done.

In this case, vehicle No. 6 will be selected because its speed is less than the other two vehicles.

Table (4) Selection of Appropriate Vehicle

\begin{tabular}{|c|c|c|c|c|c|c|}
\hline \multicolumn{7}{|c|}{ Finally we are Getting VM with minimum Speed: } \\
\hline & ID & Speed & Zone & Xa & Data & Mig_Req \\
\hline 6 & 6 & 26 & 1 & 35 & 0 & 1 \\
\hline \multicolumn{7}{|c|}{ Finally Migration VM in DB is: } \\
\hline 6 & 6 & 26 & 1 & 35 & $\begin{array}{c}\text { Data of VM } \\
\text { in 1 DB }\end{array}$ & 1 \\
\hline \\
$\begin{array}{c}\text { Where We Have 8 VM in the street, we get: } \\
\text { Number of successful migration to VM is: } 1 \\
\text { Number of migration to RUS is: 0 }\end{array}$ \\
\hline
\end{tabular}

Table (4) shows the appropriate vehicle that is chosen for the migration as well as the number of successful to destination vehicle (VCd) or To RSU.

Table (5) number of Successful migrate to VM or RSU. If there are $12 \mathrm{VMs}$ in the street, we get:

Successful migration to $\mathrm{VM}=95$

Migration to $\mathrm{RSU}=5$

If there are $8 \mathrm{VMs}$ in the street, we get:

Successful migration to $\mathrm{VM}=91$

Migration to $\mathrm{RSU}=9$

If there are $4 \mathrm{VMs}$ in the street, we get:

Successful migration to $\mathrm{VM}=74$

Migration to RSU $=26$

Table (5) shows the number of successful migration to (VCd) when different vehicles in street, and migrate to RSU.

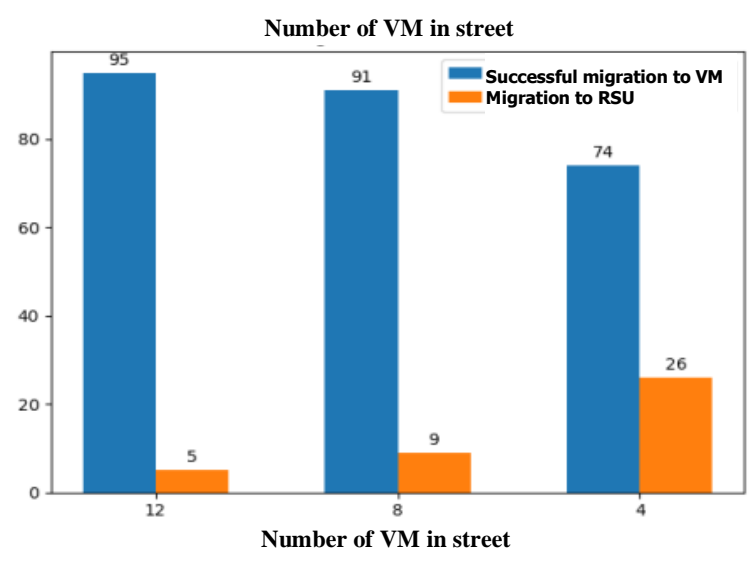

Figure 5: shows the percentage of successful migration to VM or RSU

When there are many vehicles in the service zone, the possibility of deportation to other vehicles is large because there are many options for deportation, but when there are not many vehicles in the service zone, there will be no vehicles suitable for the VM migration and will be migration to RSU. Figure 5 shows the percentage of successful migration to $\mathrm{VM}$ and also the migration to RSU.

\section{CONCLUSION}

In this paper, the challenges of exploiting cloud computing in vehicular networks were first discussed. Then, a hierarchical architecture for cloud-based vehicular networks that facilitates the sharing of computational resources, storage resources and bandwidth resources among vehicles was presented. Furthermore, it was focused on continuity of service in the vehicle for that VM migrate was proposed to avoid data loss during service interruption and avoid wasting time when re-selecting other vehicle and establishing a connection with it.

To complete the migration, an algorithm was proposed to choose a vehicle that is identical to the conditions and an algorithm to perform the migration process. By simulating the two algorithms, the remaining time of the vehicle in the service zone has been calculated and the migration process has been done.

Finally, the results showed that the migration process led to data preservation and the service was not interrupted during the use by the user

\section{REFERENCES}

[1] Mell P, Grance T. The NIST Definition of Cloud Computing. Maryland, US: The National Institute of Standards and Technology; 2011.

[2] Peter Mell and Timothy Grance. The nist definition of cloud computing (draft). NIST special publication, 800(145):7, 2011

[3] S.BITAM, A.MELLOUK, S.ZEADALLY, VANET-cloud: A generic cloud computing model for vehicular Ad Hoc networks ", IEEE Wireless Communications 22(1):96-102 - February 2015

[4] M. Abuelela and S. Olariu, "Taking VANET to the clouds", In Proceedings of ACMMoMM, Paris, France, November 2010.

[5] S. Arif, S. Olariu, J.Wang, G. Yan,W. Yang, and I. 
Khalil, "Datacenter at the airport: Reasoning about Time-Dependent Parking Lot Occupancy", IEEE Transactions on Parallel and Distributed Systems 23(11):2067-2080, 2012.

[6] M. Eltoweissy, S. Olariu and M. Younis, "Towards autonomous vehicular clouds", In Proceedings AdHocNets, Victoria, BC, Canada, August 2010.

[7] S. Olariu, I. Khalil, and M. Abuelela, "Taking VANET to the Clouds", International Journal of Pervasive Computing and Communications 7(1):721, 2011.

[8] S. Olariu, M. Eltoweissy, and M. Younis. Towards autonomous vehicular clouds, ICST Transactions on Mobile Communications and Computing 11(7-9):111,2011

[9] M. Whaiduzzamana, M. Sookhaka, A. Gania and R. Buyyab, "A Survey on Vehicular Cloud Computing," Journal of Network and Computer Applications, 2013.

[10] R. Hussain, J. Son, H. Eun, S. Kim and H. Oh, "Rethinking Vehicular Communications: Merging V ANET with Cloud Computing," in Proc. Of the 4th IEEE International Conference on Cloud Computing Technology and Science, Taipei, Taiwan pp. 606609, 2012.

[11] Oanh Tran Thi Kim, Nguyen Dang Tri, VanDung Nguyen, and Choong Seon Hong, "Addressing Virtual Machine Migration Problems in Cloud Based Vehicular Networks", Korea Computer Engineering Conference ,2015.

[12] F Ahmad, M Kazim, A Adnane , A Awad " Vehicular Cloud Networks: Architecture, Applications and Security Issues"2015 IEEE/ACM 8th International Conference on Utility and Cloud Computing, pp.571-576, DOI 10.1109/UCC.2015.101

[13] T. Refaat, B. Kantarci and H. Mouftah, "Virtual machine migration and management for vehicular clouds", Vehicular Communications, vol. 4, pp. 47$56,2016$.

[14] Rong Yu, Yan Zhang, Huimin Wu, Periklis Chatzimisios, and Shengli Xie, "Virtual Machine Live Migration for Pervasive Services in CloudAssisted Vehicular Networks" 8th International Conference on Communications and Networking in China (CHINACOM) ,pp.540.545, 2013.

[15] Rong Yu; Yan Zhang; Gjessing, S.; Wenlong Xia; Kun Yang, "Toward cloud-based vehicular networks with efficient resource management," Network, IEEE, vol.27, no.5, pp.48,55, September-October 2013.

[16] Pankajdeep Kaur and Anita Rani. "Virtual Machine
Migration in Cloud Computing". International Journal of Grid Distribution Computing Vol. 8, No.5, (2015), pp.337-342.

[17] D. Kapil, E. S. Pilli, and R. C. Joshi, "Live virtual machine migration techniques: Survey and research challenges," in 2013 3rd IEEE International Advance Computing Conference (IACC), 2013, pp. 963-969.

[18] Anna Maria Vegni, Mauro Biagi and Roberto Cusani, 'Smart Vehicles, Technologies and Main Applications in Vehicular Ad hoc Networks' ISBN 978-953-51-0992-1, Published: February 13, 2013.

[19] M. Mishra, A. Das, P. Kulkarni, A. Sahoo, "Dynamic resource management using virtual machine migrations", IEEE Communications Magazine, vol.50, no.9, pp.34-40, 2012.

[20] S. K. Bose, S. Brock, R. Skeoch and S. Rao, "Cloudspider: Combining replication with scheduling for optimizing live migration of virtual machines across wide zone networks", in Proc. International Symp. On Cluster, Cloud and Grid Computing, pp. 13-22, 2011.

[21] K. Ye, X. Jiang, D. Huang, J. Chen, and B. Wang. "Live Migration of Multiple Virtual Machines with Resource Reservation in Cloud Computing Environments", in Proc. IEEE CLOUD, July 2011.

[22] H. Arkian, R. Atani, A. Diyanat and A. Pourkhalili, "A cluster-based vehicular cloud architecture with learning-based resource management", The Journal of Supercomputing, vol. 71, no. 4, pp. 1401-1426, 2015.

[23] Wenshuang Liang, Zhuorong Li, Hongyang Zhang, Shenling Wang, and Rongfang Bie, "Vehicular Ad Hoc Networks: Architectures, Research Issues, Methodologies, Challenges, and Trends," International Journal of Distributed Sensor Networks, vol. 2015, Article ID 745303, 11 pages, 2015.

[24] Hong Yao, Changmin Bai, Deze Zeng, Qingzhong Liang, Yuanyuan Fan: Migrate or not? Exploring virtual machine migration in roadside cloudlet-based vehicular cloud . Concurrency and Computation: Practice and Experience 27(18): 5780-5792 (2015).

[25] Ahmad M. Mustafa, Omar M. Abubakr, Omar Ahmadien, Ahmed Ahmedin, Bassem Mokhtar: Mobility Prediction for Efficient Resources Management in Vehicular Cloud Computing .MobileCloud 2017: 53-59.

[26] Anita Choudhary, Mahesh Chandra Govil, Girdhari Singh, Lalit K. Awasthi, Emmanuel S. Pilli, Divya Kapil: A critical survey of live virtual machine migration techniques. J. Cloud Computing 6: 23 (2017). 